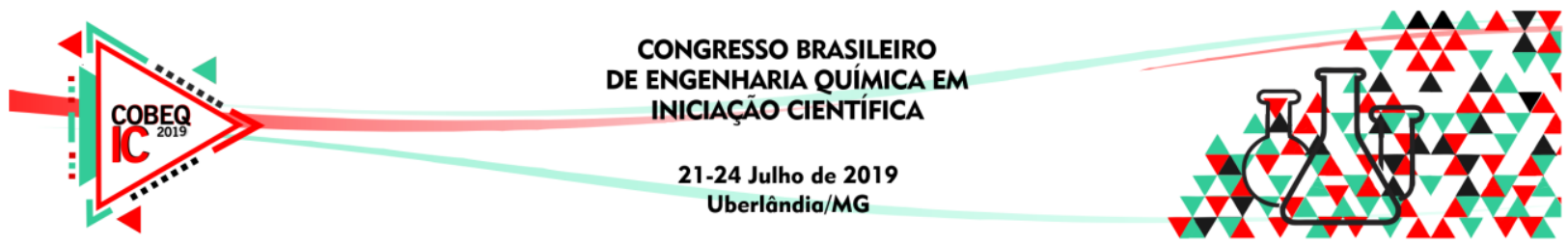

\title{
COMPARAÇÃO DA PRODUÇÃO DE L-ASPARAGINASE POR FERMENTAÇÃO EM ESTADO SÓLIDO COM FARELO DE ARROZ E ESPUMA DE POLIURETANA
}

\author{
N. V. SILVA ${ }^{1}$, W. F. VIEIRA ${ }^{1}$ e U. C. FILHO $^{1}$ \\ ${ }^{1}$ Universidade Federal de Uberlândia, Faculdade de Engenharia Química \\ E-mail para contato: nycolevir@hotmail.com
}

\begin{abstract}
RESUMO - As enzimas L-asparaginase é produto de grande destaque e aplicação chave nas indústrias alimentícias e farmacêuticas, o que justifica a busca por processos mais eficientes na produção das enzimas. Neste trabalho a comparação da produção de L-asparaginase de Penicillium sp. LAMAI 505 em fermentação submersa (FS) e fermentação em estado sólido (FES), foram analisadas e otimizadas, possibilitando um aumento de 128 vezes na produção de Lasparaginase em FES comparado a FS, e, no comparativo dos suportes sólidos houve diferença de 2,8 vezes na produção.
\end{abstract}

\section{INTRODUÇÃO}

A L-asparaginase é uma enzima encontrada em diversos seres vivos, tais como: animais, bactérias e fungos. É um importante produto utilizado na indústria alimentar, atua na prevenção de acrilamida, produto tóxico gerado pelo processamento de alimentos a altas temperaturas, e, na indústria farmacêutica como produto chave na obtenção de medicamentos utilizados no tratamento de doenças hematopoéticas e câncer, por exemplo Leucemia Linfoblástica Aguda (LLA). (HUANG et al, 2014).

Atualmente, a L-asparaginase terapêutica é produzida através do cultivo de enterobactérias Escheria coli ou Erwinia chrysanthemi em fermentação submersa (FS). Porém, a L-asparaginase bacteriana tem causado efeitos colaterais aos pacientes, causando hipersensibilidade e inativação imunológica. Mediante a este fato, a produção de Lasparaginase por fungos filamentosos em fermentação em estado sólido (FES) é uma opção vantajosa, pois reduz a imunogenicidade e diminui os custos de processo (DANGE; PESHWE, 2015).

A FES se destaca pelo baixo grau de investimento, alto rendimento, pouca utilização de água, além da boa adaptação dos fungos filamentosos nos suporte sólidos, quando comparado a FS (THOMAS; LARROCHE; PANDEY, 2013). A FES permite o uso de resíduos agroindustriais como suportes sólidos, devido ao baixo custo e composições nutricionais das biomassas, no entanto o uso de suporte inerte se destaca por possibilitar uma melhor adaptação do fungo no suporte, aumentar a produção e facilitar etapas futuras de separação do produto nobre desejado (KUMAR; DASU; PAKSHIRAJAN, 2010). 


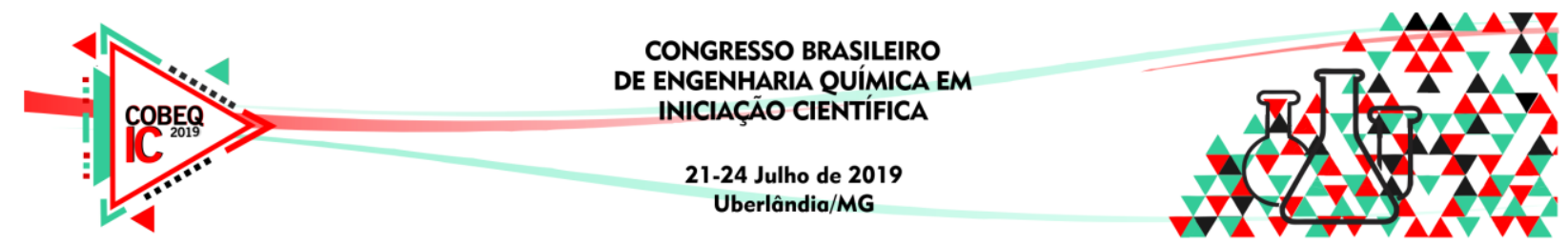

Neste trabalho a produção de L-asparaginase foi realizada por meio do Penicillium sp. LAMAI 505 em FES e FS, foi avaliada a composição de meio nutritivo, os suportes sólidos de farelo de arroz e espuma de poliuretana e o tempo de fermentação.

\section{MATERIAIS E MÉTODOS}

O presente trabalho foi desenvolvido nas dependências do laboratório de pesquisa do Núcleo de Bioquímica (NUCBIO) da Faculdade de Engenharia Química (FEQUI) da Universidade Federal de Uberlândia (UFU).

\subsection{Microrganismo}

O fungo Penicillium sp. LAMAI 505 utilizado na produção de L-asparaginase, foi obtido da Central de Recursos Microbianos do Instituto de Biotecnologia da UNESP (CRM UNESP). Afim de cultivar o microrganismo foi utilizado uma solução nutritiva Czapek, como mostra a Tabela 1 .

Tabela 1 - Composição da solução nutritiva Czapek.

\begin{tabular}{|l|c|c|}
\hline Composto & Fórmula & $\begin{array}{c}\text { Concentração } \\
(\mathrm{g} / \mathrm{L})\end{array}$ \\
\hline \hline Nitrato de sódio & $\mathrm{NaNO}_{3}$ & 2,00 \\
\hline $\begin{array}{l}\text { Fosfato de } \\
\text { potássio }\end{array}$ & $\mathrm{K}_{2} \mathrm{HPO}_{4}$ & 1,00 \\
\hline $\begin{array}{l}\text { Sulfato de } \\
\text { magnésio }\end{array}$ & $\mathrm{MgSO}_{4}$ & 0,50 \\
\hline $\begin{array}{l}\text { Cloreto de } \\
\text { potássio }\end{array}$ & $\mathrm{KCl}$ & 0,50 \\
\hline Sulfato ferroso & $\mathrm{FeSO}_{4}$ & 0,01 \\
\hline Sacarose & $\mathrm{C}_{12} \mathrm{H}_{22} \mathrm{O}_{11}$ & 30,00 \\
\hline Agar ${ }^{\text {a }}$ & - & 20,00 \\
\hline
\end{tabular}

Nota: ${ }^{a}$ A adição de Agar representa a solução nutritiva Czapek em estado sólido utilizada no cultivo do Penicillium sp. LAMAI 505.

\subsection{Preparação do inoculo}

O Penicillium sp. LAMAI 505 foi cultivado primeiramente, em placa petri com solução nutritiva de Czapek sólido $\left(72 \mathrm{~h}, 25^{\circ} \mathrm{C}\right)$, Tabela 1 . Em seguida foi realizada a etapa de crescimento celular, onde o fungo foi transferido da placa petri para o erlenmeyer de $250 \mathrm{~mL}$ contendo $100 \mathrm{~mL}$ de solução nutritiva Czapek (sem Agar, $72 \mathrm{~h}, 25{ }^{\circ} \mathrm{C}$ ), devidamente esterilizadas a $121^{\circ} \mathrm{C}$ por 20 minutos, e levada a massa agitadora a $150 \mathrm{rpm}$. Após esta etapa as células do Penicillium sp. foram separadas por centrifuga $\left(25^{\circ} \mathrm{C}, 5000 \mathrm{rpm}\right)$ e utilizadas em diferentes condições operacionais.

\subsection{Fermentação submersa e fermentação estado sólido}

A produção de L-asparaginase de Penicillium sp. LAMAI 505 em FS e FES, foi analisada em etapas distintas, comparando as condições de meio nutritivo, suportes sólidos e tempo de fermentação. A Tabela 2 mostra os diferentes ensaios experimentais realizados e as condições nutritivas usadas na produção de L-asparaginase de cada ensaio experimental. 


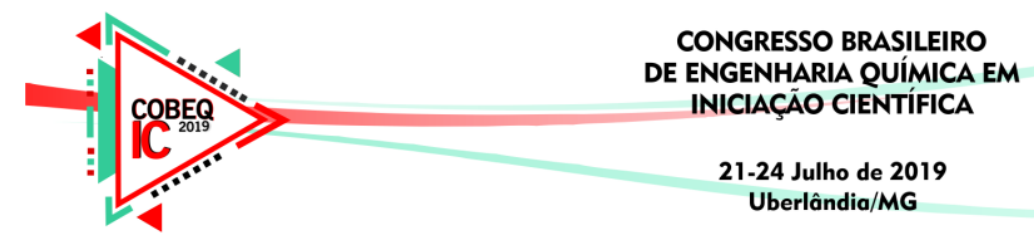

Uberlândia/MG

\begin{tabular}{|c|c|c|}
\hline Ensaios $^{\mathrm{a}, \mathrm{b}}$ & $\begin{array}{c}\text { Asparagina } \\
(\mathrm{g} / \mathrm{L})\end{array}$ & $\begin{array}{c}\text { Glicose } \\
(\mathrm{g} / \mathrm{L})\end{array}$ \\
\hline \hline 1 & 1,0 & 4,0 \\
\hline 2 & 1,0 & 10,0 \\
\hline 3 & 1,0 & 16,0 \\
\hline 4 & 5,0 & 4,0 \\
\hline 5 & 5,0 & 10,0 \\
\hline 6 & 5,0 & 16,0 \\
\hline 7 & 10,0 & 4,0 \\
\hline 8 & 10,0 & 10,0 \\
\hline 9 & 10,0 & 16,0 \\
\hline
\end{tabular}

Tabela 2 - Diferentes condições de meio nutritivo usando na produção de L-asparaginase fúngica.

Nota: ${ }^{\text {a }}$ Sais minerais ilustrado na Tabela $1 ;{ }^{\mathrm{b}}$ Os ensaios foram realizados em triplicata $\mathrm{a} 25^{\circ} \mathrm{C}$ e $72 \mathrm{~h}$ de fermentação.

A Tabela 3 mostra as 12 diferentes condições experimentais de concentração de asparagina, suportes sólidos e proporção de líquido no suporte sólido adotadas na produção de L-asparaginase por FES.

Tabela 3 - Diferentes condições de produção de L-asparaginase em FES com suportes sólidos de espuma de poliuretana e farelo de arroz.

\begin{tabular}{|c|c|c|c|}
\hline Ensaios $^{\mathrm{b}}$ & $\begin{array}{c}\text { Meio } \\
\text { sólido }^{\text {a }}\end{array}$ & $\begin{array}{c}\text { Asparagina } \\
(\mathrm{g} / \mathrm{L})\end{array}$ & $\begin{array}{c}\text { Proporção } \\
\text { de líquido } \\
\text { do suporte } \\
\text { sólido } \\
(\% \text { v/v })\end{array}$ \\
\hline \hline 1 & $\mathrm{~A}$ & 0,0 & 25,0 \\
\hline 2 & $\mathrm{~A}$ & 0,0 & 50,0 \\
\hline 3 & $\mathrm{~A}$ & 0,0 & 75,0 \\
\hline 4 & $\mathrm{~A}$ & 1,0 & 25,0 \\
\hline 5 & $\mathrm{~A}$ & 1,0 & 50,0 \\
\hline 6 & $\mathrm{~A}$ & 1,0 & 75,0 \\
\hline 7 & $\mathrm{E}$ & 0,0 & 25,0 \\
\hline 8 & $\mathrm{E}$ & 0,0 & 50,0 \\
\hline 9 & $\mathrm{E}$ & 0,0 & 75,0 \\
\hline 10 & $\mathrm{E}$ & 1,0 & 25,0 \\
\hline 11 & $\mathrm{E}$ & 1,0 & 50,0 \\
\hline 12 & $\mathrm{E}$ & 1,0 & 75,0 \\
\hline
\end{tabular}

Nota: A - Suporte sólido de farelo de arroz; E - Suporte sólido inerte de espuma de poliuretana; ${ }^{\text {a }}$ Sais minerais ilustrado na Tabela $1 .{ }^{\mathrm{b}}$ Os ensaios foram realizados em triplicata a $25^{\circ} \mathrm{C}$ e $72 \mathrm{~h}$ de fermentação.

\subsection{Método de análise de L-asparaginase}

A atividade da L-asparaginase foi determinada segundo Drainas et al., (1997), onde 0,5

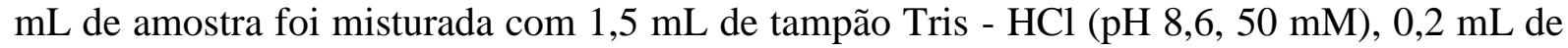
L-asparagina $(100 \mathrm{mM})$ e $0,2 \mathrm{~mL}$ de hidroxilamina $(1 \mathrm{M})$ é incubada (30 minutos, $\left.37^{\circ} \mathrm{C}\right)$. Após a reação é terminada com adição de $0,5 \mathrm{~mL}$ da solução de cloreto férrico $\mathrm{TCA} / \mathrm{HCl}$ e 


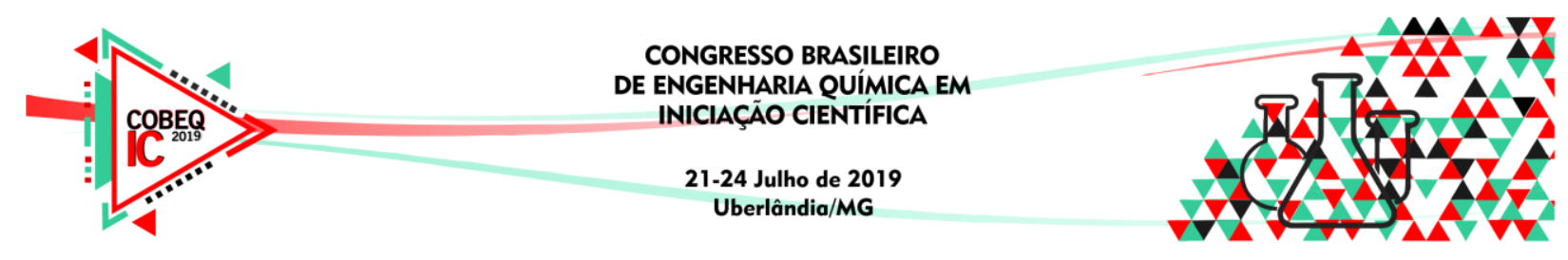

centrifugada por 10 minutos (10000g, Avanti J-26XP Series) e em seguida o teor de ácido $\beta$ hidroxilamico é obtido por espectrofotometria $(500 \mathrm{~nm}$, espectrofotômetro UV-MINI-1240) utilizando a Equação 1.

$\lambda\left(\frac{U}{k g}\right)=1000 *\left(\frac{\delta * \operatorname{Vol}_{E x t} * F_{\text {Diluicão }}}{\operatorname{Vol}_{\text {Amostra }} * 0,4 * \theta * m_{\text {Sólido }}}\right)$

Nota: $\delta$ - Leitura no espectrofotômetro, $V o l_{E x t}$ - Volume de extração, $F_{\text {Diluição }}$ Fator de diluição, $V o l_{\text {Amostra }}$ - Volume da amostra, $\theta$ - tempo de reação em banho maria e $m_{S^{\prime} \text { lido- }}$ massa de suporte sólido

\section{RESULTADOS E DISCUSSÃO}

A Figura 1 mostra a produção de L-asparaginase de Penicillium sp. LAMAI 505 em FS e FES, utilizando diferentes composições nutritivas de glicose e L-asparagina. Pode-se observar que as atividades máximas de L-asparaginase obtidas foram de $3,85 \mathrm{U} / \mathrm{kg}$ ( $1,0 \mathrm{~g} / \mathrm{L}$ de Lasparagina e 10,0 g/L de glicose) em FS e 493,1 U/kg ( 5,0 g/L de L-asparagina e 10,0 g/L de glicose), gerando um aumento de 128 vezes na atividade de L-asparaginase em FES comparado a FS. Além disso, o Penicillium sp. se mostrou capaz de produzir a L-asparaginase, visto que na literatura tem-se que Janakiraman e Meghavarnam (2017), em FES com Fusarium culmorum (ASP-87), encontraram a atividade de 1250,0 U/kg em biomassa de arroz. Considerando os resultados apresentados pela Figura 1, a FES foi selecionada para próxima etapa.

Figura 1 - Produção de L-asparaginase em fermentação submersa (FS) e fermentação em estado sólido (FES) com diferentes composições de meios nutritivos.

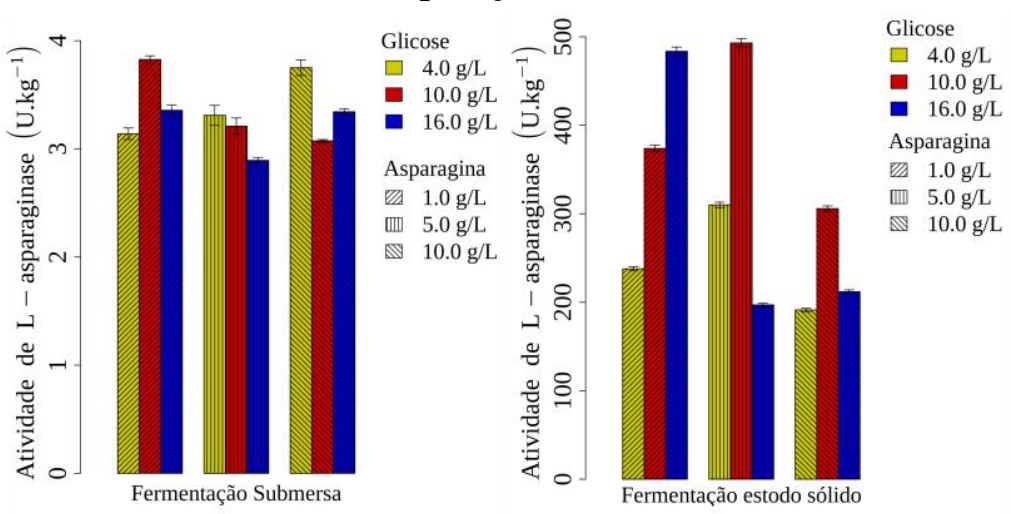

Nota: A fermentação em estado sólido (FES) foi realizada utilizando farelo de arroz como suporte sólido.

A Figura 2 mostra a produção de L-asparaginase de Penicillium sp. LAMAI 505 em FES com suporte inerte de poliuretana (Figura 2, a) e biomassa de arroz (Figura 2, b), em diferentes condições operacionais e composições de meios nutritivos (concentração de glicose fixa em 10,0 g/L). Pode-se observar que fungo produz a enzima de forma extracelular e intracelular, sendo que na FES com suporte inerte a produção extracelular se destacou, enquanto na FES com farelo de arroz a produção intracelular foi o destaque. As atividades máximas de L-asparaginase obtidas foram de 1516,7 U/kg em FES com espuma de poliuretana e 533,7 U/kg 


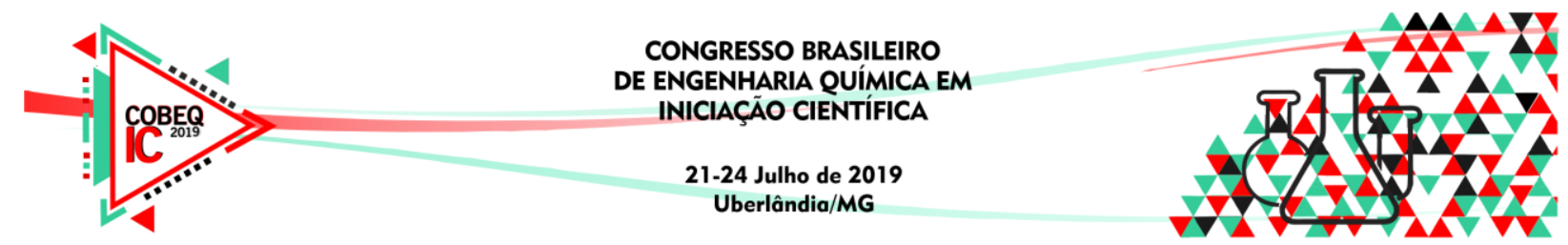

em FES com farelo de arroz, sendo que a FES com suporte inerte de espuma de poliuretana aumentou 2,8 vezes a atividade de L-asparaginase comparado ao suporte de farelo de arroz. Considerando os resultados ilustrados na Figura 2, a FES com espuma de poliuretana foi seleciona para análise do tempo de fermentação em condições otimizadas.

Figura 2 - Comparativo da produção de L-asparaginase por FES com suportes sólidos de farelo de arroz e espuma de poliuretana.
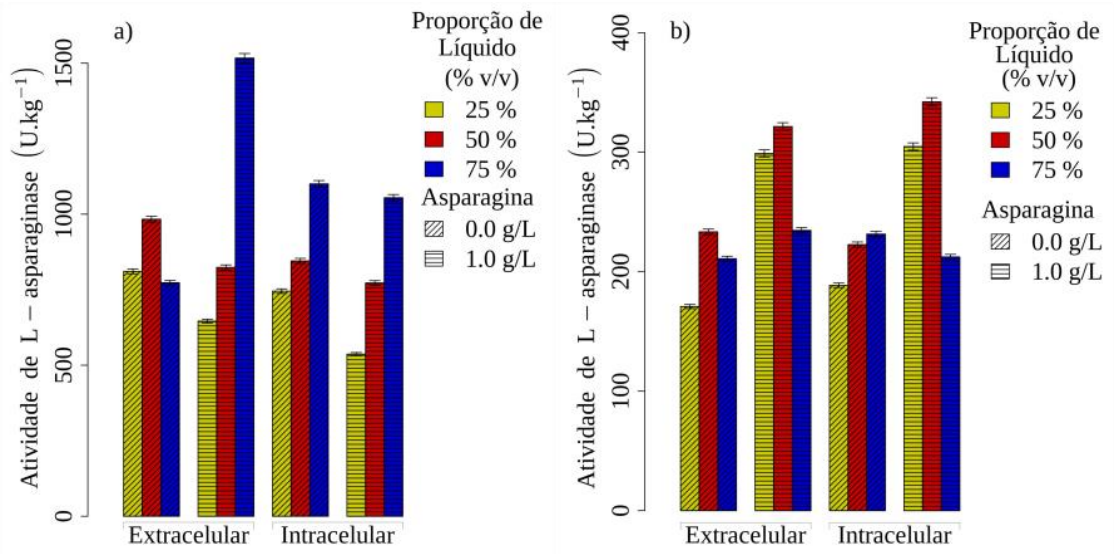

Nota: a, b - representam a produção de L-asparaginase em fermentação em estado sólido utilizando respectivamente, espuma de poliuretana e farelo de arroz como suportes sólidos.

A Figura 3 mostra a análise da produção de L-asparaginase de Penicillium sp. LAMAI 505 por FES com espuma de poliuretana. Pode-se observar que o tempo de fermentação de $48 \mathrm{~h}$ foi o mais eficiente com atividade de L-asparaginase de $1854,7 \mathrm{U} / \mathrm{kg}$, gerando um aumento de 1,22 vezes comparado com a melhor condição operacional apresentada na Figura 2 e 3,8 vezes comparando com a melhor condição operacional apresentada na Figura 1.

Figura 3 - Análise da produção de L-asparaginase por FES com espuma de poliuretana no tempo.

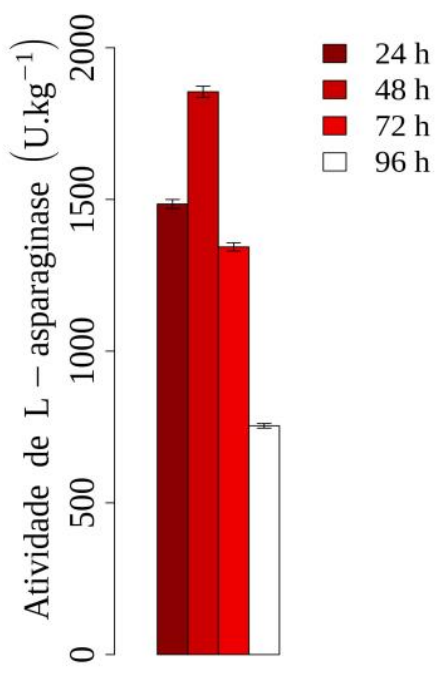




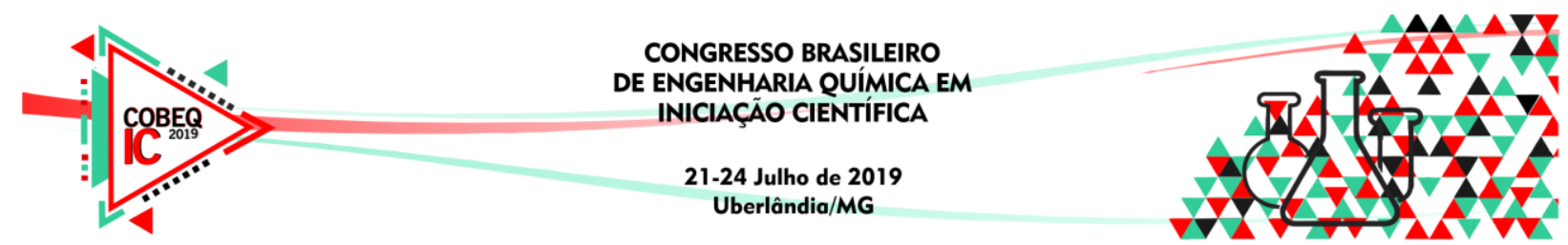

\section{CONCLUSÃO}

A produção de L-asparaginase de Penicillium sp. LAMAI 505 em fermentação submersa e fermentação em estado sólido possibilitou-se observar a capacidade do fungo filamentoso produzir a enzima de forma intracelular e extracelular. Na FES destacou-se a produção de Lasparaginase, aumentando em 128 vezes a produção em comparação a fermentação submersa. Além disso, a FES com o suporte inerte de poliuretana incrementou a produção de Lasparaginase em 2,8 vezes em relação a FES com farelo de arroz. Outro fato importante foi a redução do tempo de fermentação de $72 \mathrm{~h}$ para $48 \mathrm{~h}$, aumentando a produção de L-asparaginase em 1,22 vezes.

\section{AGRADECIMENTOS}

Os autores agradecem ao Conselho Nacional de Desenvolvimento Científico e Tecnológico (CNPq), Coordenação de Aperfeiçoamento de Pessoal de Nível Superior (CAPES), Fundação de Amparo à Pesquisa de São Paulo (FAPESP, 2012 / 08617-7) e Fundação de Amparo à Pesquisa do Estado de São Paulo. Minas Gerais (FAPEMIG, APQ 03927-16) para apoio financeiro.

\section{REFERENCIAS}

DANGE, V.; PESHWE, S. Purification and biochemical characterization of L- asparaginase from Aspergillus niger and evaluation of its antineoplastic activity. Int. J. Sci. Res., v. 4, n. 2, p. 564-569, 2015.

DRAINAS, C., KINGHORN, J. R., PATEMAN, J. A. Aspartic Hydroxamate Resistance and Asparaginase Regulation in the Fungus Aspergillus nidulans, Journal of General Microniology. 21, 493-501, 1997.

HUANG, L. et al. Biochemical characterization of a novel L-Asparaginase with low glutaminase activity from Rhizomucor miehei and its application in food safety and leukemia treatment. Applied and environmental microbiology, v. 80, n. 5, p. 1561-9, 1 mar. 2014.

KUMAR, S.; DASU, V. V.; PAKSHIRAJAN, K. Localization and production of novel 1asparaginase from Pectobacterium carotovorum MTCC 1428. Process Biochemistry, v. 45, n. 2, p. 223-229, fev. 2010.

MEGHAVARNAM, A. K., JANAKIRAMAN, S. Solid state fermentation: An effective fermentation strategy for the production of L-asparaginase by Fusarium culmorum (ASP87), Biocatalysis and Agricultural Biotechnology. 11, 124-130, 2017.

THOMAS, L.; LARROCHE, C.; PANDEY, A. Current developments in solid-state fermentation. Biochemical Engineering Journal, v. 81, p. 146-161, dez. 2013. 